\title{
Optimization of Leach Protocol in Wireless Sensor Network
}

\author{
Bhavesh Pithva \\ Dept. of Electronics \& \\ Communication Engineering C. \\ U. Shah College of \\ Engineering \& \\ TechnologyWadhvan, India
}

\author{
Kunal Pattani \\ Dept. of Electronics \& \\ Communication Engineering \\ C. U. Shah College of \\ Engineering \& Technology \\ Wadhvan, India
}

\author{
Ashish Christian \\ Dept. of Electronics \& \\ Communication Engineering \\ G. H. Patel College of \\ Engineering \& Technology \\ VallabhVidhya Nagar, India
}

\begin{abstract}
Continuous advancement in wireless communication has enabled the deployment of wireless sensor networks for environment monitoring. The use of wireless sensor networks in this type of monitoring, it is very much difficult to replace the battery of the sensor node or replace whole sensor node once they deployed. So that development the different methodology to extend the lifetime of the sensor node is must.Low Energy Adaptive Clustering Hierarchy (LEACH) is cluster based routing protocol which provides life expectancy. In this paper proposed new routing protocol Energy Efficient Hybrid LEACH (EH-LEACH) in which the threshold criteria for selection of cluster head is changed and also consider residual energy of the sensor node. The simulation results clearly indicate that proposed approach is very much energy efficient as compared to LEACH. Also EH-LEACH is sustaining his properties while varying node density. Optimization of cluster based hierarchical routing protocols is required for Better Operations.
\end{abstract}

\section{General Terms}

Two ray model, Cluster head selection algorithm, Steady state phase, Threshold Criteria,Sensor nodes.

\section{Keywords}

Wireless Sensor Network;LEACH; Lifetime; EH- LEACH.

\section{INTRODUCTION}

Recent development in the area of the networkcommunications \& sensor technologies making wide range of research area in the field of wireless sensor network A wireless sensor network is a wireless ad-hoc network having large number of small, low cost, low power and intelligent sensor nodes which monitor physical or environmental conditions such as temperature, pressure, motion etc. at different regions [3]. The sensors are randomly placed around a sensing field to collect information about their surroundings. One of the unique features that WSN is smart nodes are fitted with an onboard processor. And also Instead of sending the original data to the nodes responsible for the fusion, they use their processing abilities to locally carry out simple computations and transmit only the required and partially processed data. Because of these features WSN have potential applications and expand human ability to monitor and interact remotely with the physical world.

Network protocols must be designed to achieve fault tolerance in the presence of individual node failure due to battery operated while minimizing energy consumption. Also the limited channel bandwidth must be shared among all the nodes in the network, so routing protocols for these networks must be able to perform local collaboration to reduce bandwidth requirements \& energy consumptions. Eventually, the data being sensed by the nodes in the network must be transmitted to a control center or base station, where the enduser can access the data. There are many possible network models for these networks. In this work, consideration of all nodes in the network are homogeneous and energy constrained [1].

Nodes are battery operated and have low computational capacity, so these nodes remain fixed once they are deployed in the application environments. Also, cost of battery replacement of the nodes are very expensive.Hence, network lifetime enhancement is a challenging issue and hot research area for the researchers. Also energy required for communication is very high compared to computation [3]. Hence, communication between the nodes must be minimized to prolong the network lifetime.

Thus improvement in energy efficiency is one of the hottest issues and designing power efficient protocols are difficult for improve the lifetime. Clustering the network is an efficient way to organize WSNs [4]. A cluster head is responsible for passing any information collected by the nodes in its cluster and may aggregate data before transmitting it to the base station. However, the function of aggregation required higher energy. One of the most interesting \& bench mark clustering mechanisms - LEACH addresses this by probabilistically rotating the role of cluster head among all nodes [2]. However the optimality of the network performance depends upon how wisely the selection of the cluster head occurs. The objective of the paper is to develop an algorithm that will calculate the optimal probability with which a node should become a cluster head in order to keep the energy consumption at its minimal requirement.

\section{CHANNEL PROPAGATION MODEL AND RADIO ENERGY DISSIPATION MODEL}

For the wireless communication it usesthe wireless channel, in which the electromagnetic wave propagation can be modeled as falling off as a power law function of the distance between the transmitter and receiver. Both free space model which considered direct line-of-sight and two-ray ground propagation model which considered ground reflected signal also, were considered depending upon the distance between transmitter and receiver [5]. If the distance is less than crossover, free space model used and If the distance is greater than crossover, two-ray ground propagation model is used. The crossover is defined as follows (1): 


$$
d_{\text {crossover }}=\frac{4 \pi * \sqrt{L} * h_{r} * h_{t}}{\lambda}
$$

Where, $\mathrm{L} \geq 1$ is system loss factor. hr is the height of the receiving antenna, htisthe height of the transmitting antenna and $\lambda$ is the wavelength of the carrier signal.The transmitted power is attenuated based on following (2):

$P_{r}(d)= \begin{cases}\frac{P_{t^{*} G_{t} * G_{r} * \lambda^{2}}}{(4 \pi d)^{2} * L} & \text { if } d<d_{\text {crossover }} \\ \frac{P_{t^{*} G_{t} * G_{r} * h_{t}^{2} * h_{r}^{2}}}{d^{4}} & \text { if } d \geq d_{\text {crossover }}\end{cases}$

Where, $\mathrm{Pr}$ is the received power at distance $\mathrm{d}$, $\mathrm{Pt}$ is transmitted power, Gt is gain of the transmitting antenna and $\mathrm{Gr}$ is gain of the receiving antenna.

In this research proposed radio energy model as described in[1] The radio energy model described about the radio characteristics, including energy dissipation in the transmit and receive modes. Transmitter will dissipates energy to run the radio electronics and power amplifier and receiver dissipates energy to run the radio electronics.Fig.1.shows the radio energy dissipation model. Using this radio model, to transmit k-bit message at distance $d$ the radio expends (3), (4):

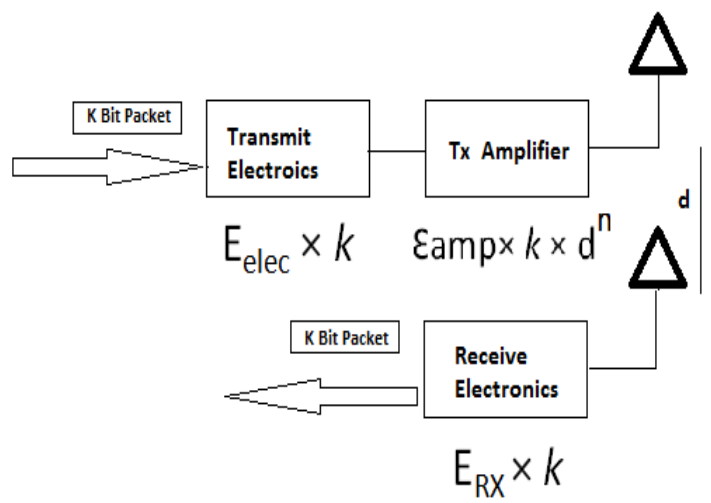

Fig.1. Radio energy dissipation model

$$
\begin{aligned}
& E_{T x}(k, d)=E_{T x-\text { elec }}(k)+E_{T x-\text { emp }}(k, d) \\
& E_{T x}(k, d) \\
& =\left\{\begin{array}{l}
E_{\text {elec }} * k+\varepsilon_{f s} * k * d^{2} \text { if } d<d_{0} \\
E_{\text {elec }} * k+\varepsilon_{m p} * k * d^{4} \text { if } d \geq d_{0}
\end{array}\right.
\end{aligned}
$$

And to receive this message, the radio expends (5), (6):

$$
E_{R x}(k)=E_{R x-\text { elec }}(k)
$$$$
E_{R x}(k)=E_{\text {elec }} * k
$$

In the simulation work it is assumed that each sensor node is enough to act as a cluster head and implements data fusion. In other words all sensor nodes have enough power to reach the sink. The algorithm also ascertains that nodes have data to send periodically. In thesimulated network, all the nodes have the same amount of initial energy capacity at starting of rounds. Hence, Developed algorithms possess node homogeneity.

\section{LOW ENERGY ADAPTIVE CLUSTERING HIERARCHY PROTOCOL}

The Low Energy Adaptive Clustering Hierarchy (LEACH) protocol combines TDMA-style contention-free communication with a clustering algorithm for wireless sensor networks. The basic operation of this protocol includes nodes which all have capabilities to elect themselves as cluster head as per threshold criteria and also role of cluster head is rotating among the nodes so that no any burden given to the any one node and life time of network is improved. A Network consists of clusters in which every cluster has single cluster head and any number of cluster members, which only communicate with their cluster head. Clustering is a popular approach for sensor networks since it facilitates data aggregation and in-network processing at the cluster head to reduce the amount of data that needs to be transmitted to the base station.

By doing so LEACH protocol improves the energy efficiency of wireless sensor networking model beyond the conventional clustering architecture; which in turn results inthe extension of the life time of proposed entire network, and this is the hot issue that is considered in the wireless sensor networking field as all the nodes are being supported by the battery.

LEACH operates in rounds consisting of two phases: a setup phase and a steady-statephase.

\subsection{Setup Phase}

Setup phase includes $\mathrm{CH}$ selection algorithm \& cluster formation algorithm. Setup phase starts with the self-election of sensor nodes to become CHs. This algorithm ensures that $\mathrm{CH}$ role rotates among sensor nodes to distribute energy among nodes. A setup up phase algorithm is shown in Fig.2.

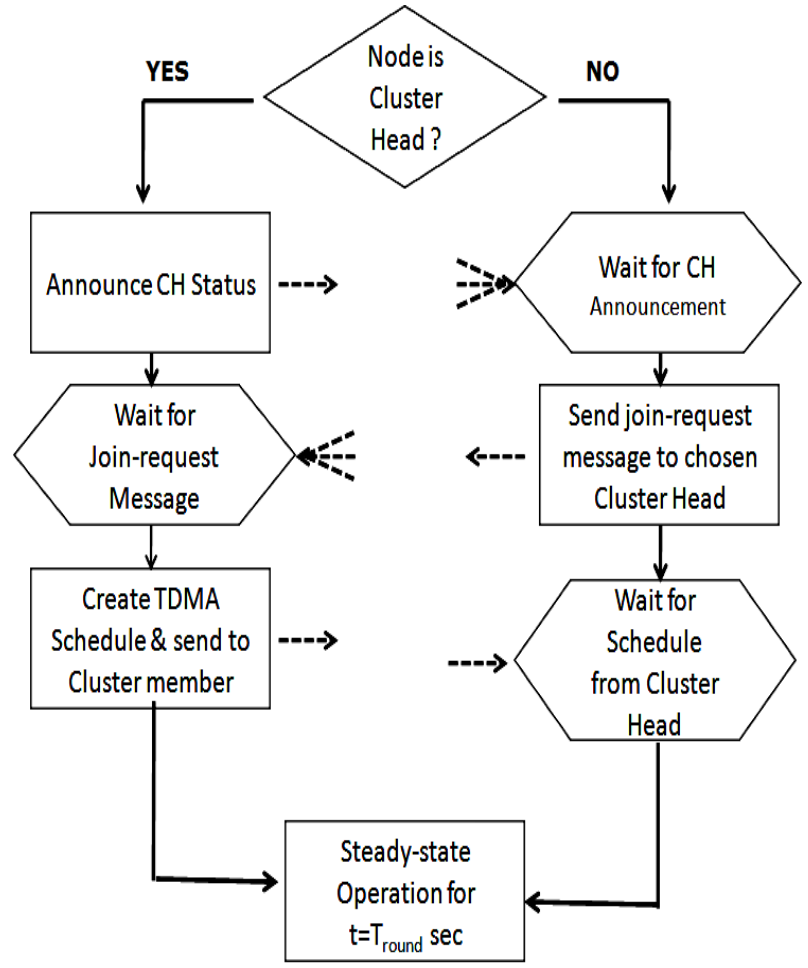

Fig.2. Setup phase algorithm[8] 


\subsubsection{Cluster Head Selection Algorithm}

The Cluster head selection algorithm is simple using random choice for $\mathrm{CH}$ selection. Since the cluster head is only responsible for coordinating cluster activity and send data to the base station, its energy requirements will be significantly large compared to other sensor nodes. So that, LEACH protocol rotates the cluster head responsibility among sensor nodes to evenly distribute the energy load. Specifically, at the beginning of a round, every sensor nodeelects itself to be a cluster head with a certain probability [2]. A node generates any random number between 0 and 1 . It then compares it with $\mathrm{CH}$ selection threshold, $\mathrm{T}(\mathrm{n})$. If this random number is less than the threshold $\mathrm{T}(\mathrm{n})$, the sensor node is a cluster head. $\mathrm{T}$ (n) is calculated as given follows (7):

$$
T(n)= \begin{cases}\frac{P}{1-P *\left(r \bmod \frac{1}{P}\right)} & \text { if } n \in G \\ 0 & \text { otherwise }\end{cases}
$$

Where $\mathrm{P}$ is the aspired percentage to become a cluster head, $\mathrm{r}$ is the current round, and $\mathrm{G}$ is the set ofnodes that have not being preferred as a cluster head in the last $1 / \mathrm{P}$ rounds (generally the percentage $\mathrm{P}$ is chosen less than or equal to10\% of the total number of nodes)[8].

\subsubsection{Cluster Formation Algorithm}

Once a sensor node has determined that it will serve as cluster head for the next round, it informs other sensor nodes of its new role by broadcasting an advertisement message using a non-persistent CSMA protocol. Once the sensor nodes receive the advertisement, they determine the cluster to which they want to belong based on the signal strength of the advertisement from the cluster heads to the sensor nodes. The sensor nodes inform the appropriate cluster heads that they will be a member of the cluster. Afterward, the cluster heads assign the time on which the sensor nodes can send data to the cluster heads based on a TDMA approach[4]. The cluster head establishes a transmission schedule for its cluster and transmits this schedule to each node in its cluster. The completion of setup phase triggers beginning of the steadystate phase.

\subsection{Steady State Phase}

During this phase, Non cluster head nodes periodically collect sensor data and transmit it to $\mathrm{CH}$ in their allocated slots. The entire steady-state operation is broken into frames which are further broken into slots of constant duration. Non cluster head nodes send collected sensor data to their respective $\mathrm{CH}$ at most once per frame during their allocated transmission slot and enter the sleep mode otherwise. Data transmissions are scheduled to avoid collisions and increase sleep time of each $\mathrm{NCH}$ node. With slots of constant duration, time to send a frame of data depends on the number of nodes in the cluster. After a certain period of time spent on the steady-state phase, the network goes into the setup phase again and enters another round of selecting cluster heads[4].

A steady state algorithm is as shown in Fig.3.

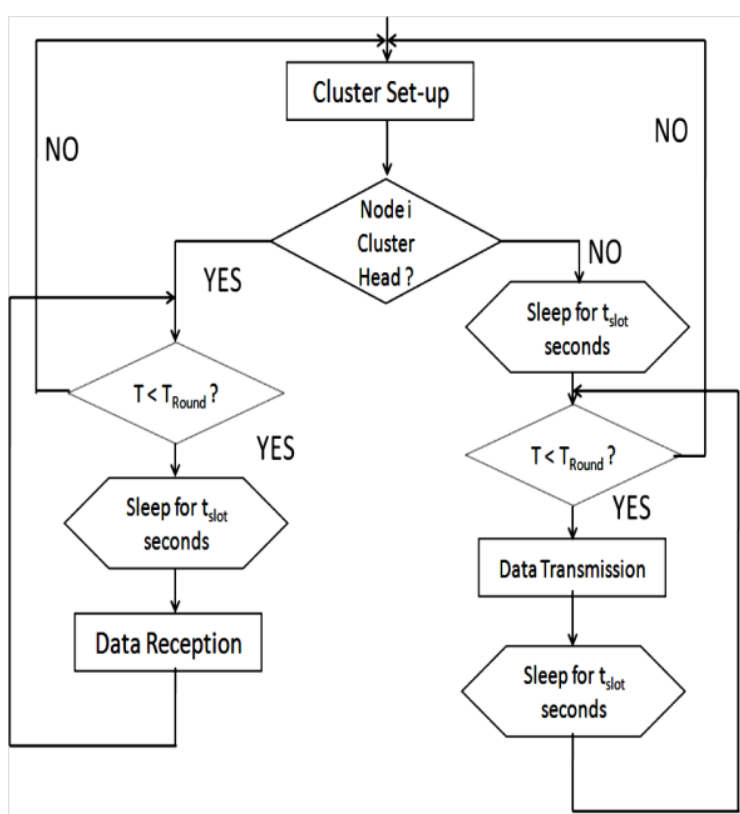

Fig.3. Steady state algorithm [8]

\section{LIFETIME PROLONGING OF LEACH}

In this proposed modification of LEACH's cluster-head selection algorithm is to reduce energy consumption where an optimal cluster head selection algorithm prolongs the lifetime of wireless sensor network based on the LEACH architecture. The major disadvantage of LEACH is that The $\mathrm{CH}$ selection algorithm does not consider node's residual energy and location with respect to $\mathrm{CH} \& \mathrm{BS}$. So this kind of $\mathrm{CH}$ selection technique is not competent enough to ensure proper $\mathrm{CH}$ selection and it is quite possible that nodes located at long distances from BS and/or the ones having less residual energy may be selected as CHs. For efficient $\mathrm{CH}$ selection, mechanisms that consider the node's distance from BS and its residual energy are required.

Proposed implementation techniques for overcome disadvantage of LEACH identified above. The EH-LEACH is proposed. Mainly to increase lifetime of network, node having more residual energy should have high probability to become the $\mathrm{CH}$. Hence proposed technique is use node's Ecurrent/Einitial for selection of $\mathrm{CH}$. The energy consumed in transmission of data from $\mathrm{CH}$ to $\mathrm{BS}$ is directly proportional to distance between them. In EH-LEACH the threshold criteria is modified according to denote in equation (8), which consider the residual energy of the sensor node \& also distance from BS.

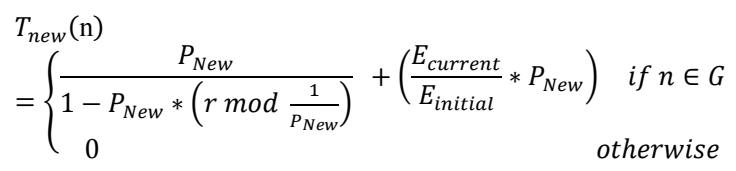

$$
P_{\text {New }}=\sqrt{\frac{n}{2 \pi}} * \sqrt{\frac{\varepsilon_{f s}}{\varepsilon_{m p}}} * \frac{X m}{d_{n B S}^{2}}
$$

Where, dnBS is distance of node from BS, Ecurrent is the current energy, and Einitial is the initial energy of sensor node, $\mathrm{Xm}$ is length of network. To decide if it is its turn to become a $\mathrm{CH}$, a node, $\mathrm{n}$, generates a random number between 0 and 1 , If number $>T_{N e w}(n)$, node becomes $\mathrm{CH}$. 
Sensor nodes elect themselves to be local cluster heads at any given time with the modified threshold Tnew (n) as per equation (8). These cluster head nodes broadcast their status to the other sensors in the network. The operation of EH$\mathrm{LEACH}$ is same as LEACH.

\section{NETWORK MODEL}

For this proposed approach, a few reasonableassumptionshave been proposed for the network model based on asfollows:

1. The base station is fixed.

2. The sensor nodes are homogeneous and energyconstrained with uniformly energy.

3. No mobility of sensor nodes.

4. All nodes are able to reach BS.

5. Nodes RAM size should be sufficient enough to store the distance ofthe nodes from Base station.

\section{SIMULATION RESULTS}

In this section presented various results have been explore the relationship between various WSN parameters and the network lifetime. In this section all simulation results are derived using MATLAB. The simulations assume the radio characteristics mentioned in Table1.A simulation has been defined the homogeneous sensor network with 50,100,200 and 500 number of sensor nodes are distributed randomly in the $100 \times 100 \mathrm{~m} 2$ area and BS at $(50,50)$.

Table 1. Simulation parameters

\begin{tabular}{|l|l|}
\hline Parameters & Value \\
\hline Nodes & Range[50-500] \\
\hline Network Size & $100 \times 100 \mathrm{mtr}$ \\
\hline BS Location & $(50,50)$ \\
\hline $\mathrm{E}_{0}$ & $0.5 \mathrm{~J}$ \\
\hline $\mathrm{E}_{\text {elec }}$ & $5 \mathrm{~nJ} / \mathrm{bit}$ \\
\hline $\mathrm{E}_{\mathrm{fs}}$ & $10 \mathrm{pJ} / \mathrm{bit} / \mathrm{m}^{2}$ \\
\hline $\mathrm{E}_{\mathrm{mp}}$ & $0.0013 \mathrm{pJ} / \mathrm{bit} / \mathrm{m}^{4}$ \\
\hline $\mathrm{E}_{\mathrm{da}}$ & $5 \mathrm{~nJ} / \mathrm{bit} / \mathrm{message}$ \\
\hline $\mathrm{d}_{0}$ & $87 \mathrm{~m}$ \\
\hline Data Packet size & $4000 \mathrm{bits}$ \\
\hline Height of Antenna(ht,hr) & $1.5 \mathrm{~m}$ \\
\hline Gain of Antenna(Gt,Gr) & 1 \\
\hline Signal Wavelength & $0.325 \mathrm{~m}$ \\
\hline Monte Carlo Iteration & 10 \\
\hline &
\end{tabular}

A packets deliver to the BS in the LEACH \& EH-LEACH is as given in Table 2. A clearly investigation has been shown that packets deliver in LEACH is very much improved in EHLEACH.

Table 2. Packets deliver to BS

\begin{tabular}{|c|c|c|}
\hline \multirow{2}{*}{$\begin{array}{c}\text { No. } \\
\text { Nodes }\end{array}$} & \multicolumn{2}{|c|}{ Packets Deliver To BS } \\
\cline { 2 - 3 } & LEACH & EH-LEACH \\
\hline $\mathrm{n}=50$ & 7247 & 113600 \\
\hline $\mathrm{n}=100$ & 12800 & 225400 \\
\hline $\mathrm{n}=200$ & 25300 & 453100 \\
\hline $\mathrm{n}=500$ & 62550 & 1111000 \\
\hline
\end{tabular}

A very much important parameter for WSN is Lifetime of the sensor node. In this simulation both LEACH and EH-LEACH have been simulated for different nodes and have been given very much improved lifetime in EH-LEACH as shown in Table 3 .

Table 3. Analyzed parameters

\begin{tabular}{|l|c|c|c|c|c|c|}
\hline \multirow{3}{*}{$\begin{array}{c}\text { Parame } \\
\text { ters }\end{array}$} & \multicolumn{2}{|c|}{ Number of nodes in wireless sensor network } \\
\cline { 2 - 7 } & \multicolumn{2}{|c|}{$\boldsymbol{N}=\mathbf{5 0}$} & \multicolumn{2}{c|}{$\boldsymbol{N = 2 0 0}$} & \multicolumn{2}{c|}{$\boldsymbol{N = 5 0 0}$} \\
\cline { 2 - 7 } & $\begin{array}{c}\boldsymbol{L E A} \\
\boldsymbol{C H}\end{array}$ & $\begin{array}{c}\boldsymbol{E H}- \\
\boldsymbol{L E A} \\
\boldsymbol{C H}\end{array}$ & $\begin{array}{c}\boldsymbol{L E A} \\
\boldsymbol{C H}\end{array}$ & $\begin{array}{c}\boldsymbol{E H}- \\
\boldsymbol{L E A} \\
\boldsymbol{C H}\end{array}$ & $\begin{array}{c}\boldsymbol{L E A} \\
\boldsymbol{C H}\end{array}$ & $\begin{array}{c}\boldsymbol{E H}- \\
\boldsymbol{L E A} \\
\boldsymbol{C H}\end{array}$ \\
\hline FND & 938 & 1632 & 1009 & 1723 & 1025 & 1618 \\
\hline TND & 1016 & 2072 & 1099 & 2026 & 1146 & 1974 \\
\hline HND & 1212 & 2328 & 1221 & 2295 & 1234 & 2320 \\
\hline
\end{tabular}

The simulation results for FND of LEACH and EH-LEACH for 100 nodes is as shown in Fig.4. Also for TND and HND is as shown in Fig.5 and Fig.6 respectively.

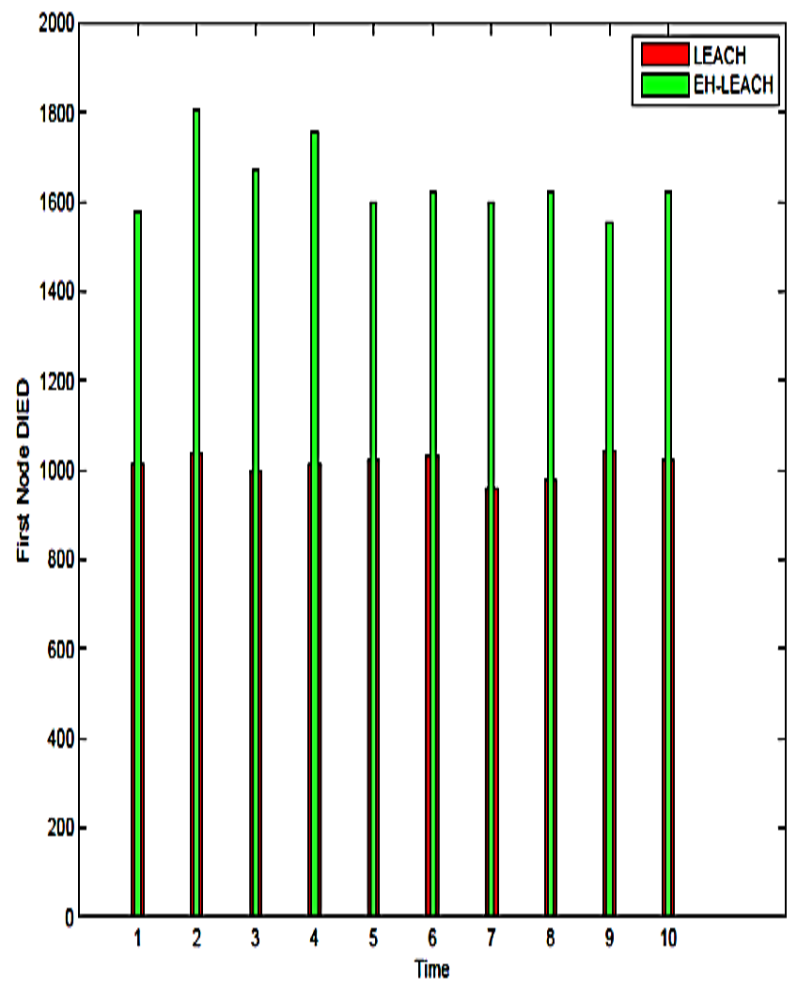

Fig.4. Life cycle comparison for FND 


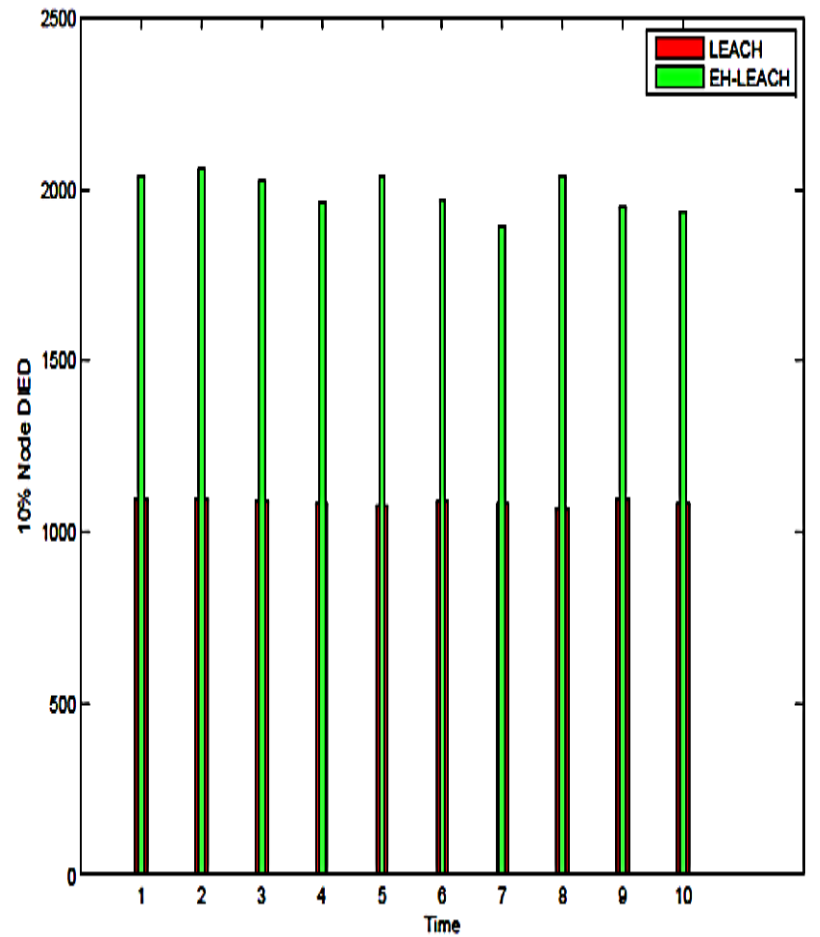

Fig.5. Life cycle comparison for TND

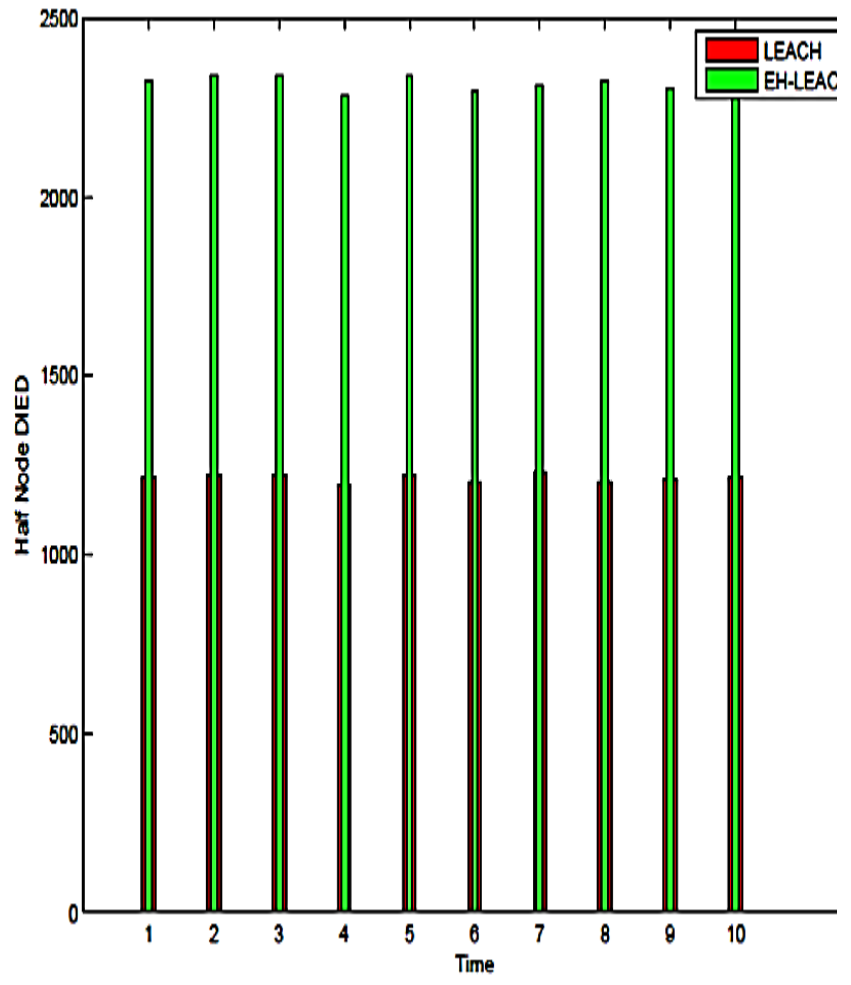

Fig.6. Life cycle comparison for HND

As Fig. 4 shows that EH-LEACH is $76 \%$ better thanLEACH with respect to First Node Died (FND).Fig.5 shows that EHLEACH is $72 \%$ better than LEACH with respect to $10 \%$ of node died (TND).Fig.6 shows that EH-LEACH is 91.6\% better than LEACH with respect to Half Node Died (HND).

\section{ACKNOWLEDGEMENTS}

Our thanks to the institutes and other experts who have contributed towards development of this research work.

\section{CONCLUSION}

In this Research paper proposed EH-LEACH protocol, provides sustain network while varying nodes. Also EHLEACH gives very much improved lifetime than LEACH and HND is very much better than LEACH. Thus EH-LEACH gives network stability and prolongs the lifetime of the network. Also in future work will be done on mobility of the base station and optimize location of BS.

\section{REFERENCES}

[1] Wendi RabinerHeinzelman, AnanthaChandrakasan, and HariBalakrishnan" Energy Efficient Communication Protocol ForwirelessMicrosensor Networks" Proceedings of the 33rd Hawaii International Conference on System Sciences, 2000.

[2] Wendi B. Heinzelman, Anantha P. Chandrakasan, AndHariBalakrishnan, An Application-Specific Protocol Architecture For Wireless MicrosensorNetworks", IEEE Trans. Wireless Commun. Vol.1, No.4, Pp660-670, 2002.

[3] Ian F. Akyildiz, Weilian Su, YogeshSankarasubramaniam, And ErdalCayirci, "A Survey On Sensor Networks", IEEE Communications Magazine, Vol. 38, No. 4, Pp. 393-422, August 2002.

[4] S. H. Gajjar, K. S. Dasgupta, S. N. Pradhan, K. M. Vala, "Lifetime Improvement Of Leach Protocol For Wireless Sensor Network", Nirma University International Conference On Engineering, Nuicone-2012, 0608december, 2012

[5] W. Heinzelman, "Application-specific protocol architectures for wireless networks," Ph.D. dissertation, Mass. Inst. Technol., Cambridge, 2000.

[6] Shuo Shi, Xinning Liu and XuemaiGu," An EnergyEfficiency Optimized LEACH-C For Wireless Sensor Networks", 7th International ICST Conference On Communications And Networking In China (CHINACOM), IEEE 2012.

[7] JiaXu,NingJin,XizhongLou,TingPeng,QianZhou, Yanmin Chen," Improvement Of Leach Protocol For Wsn", 9th International Conference On Fuzzy Systems And Knowledge Discovery ,Fskd IEEE 2012.

[8] Ashish Christian, Dr.HimanshuSoni," Lifetime Prolonging In Leach Protocol For Wireless Sensor Networks", International Conference On Intelligent Systems And Signal Processing (ISSP), 2013.

[9] Anindita Ray, Debashis De," Energy Efficient Cluster Head Selection in Wireless Sensor Network", 1st Int'l Conf. on Recent Advances in Information Technology, RAIT-2012.

[10] Holger Karl and Andreas Willig, "Protocols And Architectures For Wireless Sensor Networks".

[11] Xu-Xun Liu," A Survey on Clustering Routing Protocols in Wireless Sensor Networks", Sensors 2012. 